\title{
Comparative Studies of Electrolytes for Gold Plating
}

\author{
PAPERS AT THE INSTITUTE OF METAL FINISHING CONFERENCE
}

During the Annual Conference of the Institute of Metal Finishing, held in May at Lake Windermere, one session was devoted to the electrodeposition of gold. Three papers were presented, each in a different way discussing the relative merits and disadvantages of the number of electrolytes now available to the electroplater.

\section{Solutions for Selective Plating}

Just as the equipment used for selective gold plating consists of highly specialised machines specifcally designed for each type of application, the electrolytes must also be formulated to suit the high speed plating conditions, and the choice of solutions to be used in these sophisticated machines was discussed in a paper by F. I. Nobel and R. T. Hill of Lea-Ronal. After describing their laboratory methods of developing electrolytes-initially relying largely upon the Hull cell as a research tool-the authors emphasised that for the development of high speed plating operations new plating cells which more closely simulated operating conditions were required. Even when using such experimental cells it was found that final adjustments of the solution chemistry and operating parameters must still be carried out on the full size plating equipment in order to achieve the desired results, as there are always differences to be encountered in solution flow, jet speed and anode conditions.

Soft pure gold deposits for the semiconductor industry form the most important application of selective plating. Here the deposits are needed for the alloying, bonding and sealing operations, and must be able to withstand temperatures up to 400 or $500^{\circ} \mathrm{C}$ for several minutes and to form a reliable bond. The authors consider that no one gold plating solution, used in different types of spot plating machines, can produce gold deposits that meet all requirements, and in the paper they divide the solutions used into three categories depending upon the current density range to be used.

In the high current density range, 50 to $200 \mathrm{~A} / \mathrm{dm}^{2}$, most of the gold solutions have a high metal concentration, ranging from 10 to $40 \mathrm{~g} / \mathrm{l}$. The higher the gold content the higher is the cathode efficiency and the permissible current density in any given machine. In modern spot plating equipment one micron of gold can readily be achieved in one second, but when plating at these high speeds the bath is subject to rapid changes and must be formulated to take account of these. Free cyanide tends to build up; the use of insoluble platinum anodes and the high anode current densities maintain strong oxidising conditions in the solution which preclude or limit the use of any reagent that can readily undergo oxidation, while the high operating temperatures-generally in the range 70 to $80^{\circ} \mathrm{C}$-mean that the solution must be extremely stable.

In the medium range, from 10 to $50 \mathrm{~A} / \mathrm{dm}^{2}$, while the same conditions pertain, an additional factor is the avoidance of contamination by the co-deposition of metallic impurities such as copper, iron, nickel and cobalt. This problem can be overcome by the addition of a chelating agent capable of chelating these impurities, but one that must be stable under the plating condition, or by precipitating the contaminants. Alternatively, solutions have to be developed that are far less aggressive to the basis metal. These solutions generally operate with reduced gold concentrations of 6 to $10 \mathrm{~g} / 1$ and do not contain chelating agents.

Gold solutions designed to operate in the range 1 to $10 \mathrm{~A} / \mathrm{dm}^{2}$ are generally used in equipment that does not have the extremes of solution agitation characteristic of jet plating machines and are mostly similar to those used in conventional rack plating, but the speed of deposition is increased by raising the temperature, increasing the gold concentration, or by the use of additional agents that improve cathode efficiency and permit of somewhat higher current densities. The choice of electrolyte formulation is wider for this current density range since the extremes of anode current density and temperature are not in question.

The selective plating of nickel- or cobalt-hardened golds is not yet so generally employed as for pure gold, but is growing in the electrical contact field, and it is now possible to plate at very high speeds, up to $60 \mathrm{~A} / \mathrm{dm}^{2}$, and to obtain deposits that will pass all the tests for hardness, purity and porosity that are imposed upon rack or barrel plated hard golds. To achieve these high speeds the gold content is raised to about 10 or $15 \mathrm{~g} / 1$, the cobalt or nickel content of the solution is increased sufficiently to yield 0.2 per cent in the deposit, and the use of stable chelating agents for cobalt or nickel are required. Further, the operating temperature is raised and the solution is agitated rapidly with vigorous pumping. 
Although metallic impurities are not as critical as when plating with pure gold it is still important to prevent the nickel content becoming excessive.

Where a ductile hard gold deposit is required, it can be achieved by modifications to pure gold electrolytes, generally by raising the operating temperature and the gold content, largely on account of the high degree of solution movement at the cathode surface. Contamination limits are considerably higher in these hard golds, chiefly used in the form of electrical contacts and not for semiconductor manufacture.

\section{Cyanide or Sulphite Processes}

For something like 15 years two entirely different gold plating systems have been commercially available, the old established cyanide based electrolytes and their more recent modifications on the one hand, and the newer sulphite based solutions on the other. A detailed review of their respective advantages and disadvantages was presented in a paper by $D$. $R$. Mason and A. Blair of Engelhard Industries Limited, the comparisons being confined both to industrial uses and to relatively pure gold deposits.

Three types of cyanide based processes were discussed, the alkaline electrolytes with no base metal additions, the neutral cyanides, and the acid cyanides with small concentrations of base metals, by comparison with alkaline sulphite electrolytes with no base metal additives but containing trace quantities of arsenic. The $\mathrm{pH}$ range in which the cyanide electrolytes can be operated is much wider than for the sulphite solutions, although some investigators have shown that by the addition of a stabilising agent such as 1.2 diamino ethane the sulphite complex can be stabilised down to $\mathrm{pH} 4.7$.

Sulphite processes yield the most ductile and the hardest deposits, but as was demonstrated by Souter their poor performance in terms of increasing contact resistance and wear have rendered them unacceptable for use on connectors.

A considerable proportion of this paper dealt with the compatibility of gold plating solutions with the resists used in processing printed circuits, but direct comparison of acid gold, neutral gold and alkaline sulphite baths produced, with but a few exceptions, only slightly differing results.

In discussing the applications of the four types of electrolytes the authors referred to printed circuits for which acid cyanide golds predominate, to connectors, also mainly plated from acid cyanides, to semiconductors, where neutral cyanides are generally in use, soft deposits of pure gold, and to reed relays where again acid cyanide golds, sometimes with cobalt but more frequently nickel, have been found to be most acceptable.
In the authors' opinion, for every sulphite gold process being used today there must be more than 20 cyanide gold processes, but they nevertheless drew attention to the advantages of the sulphite electrolytes in some applications where their good throwing power and high cathode efficiency would be valuable, or where they could be employed in duplex systems, where they offer a good levelling, self-brightening undercoat.

\section{Comparison with the Platinum Metals}

That the overall reliability of an electronic system depends as much on its interconnections as on any active solid state device was emphasised by $\mathrm{Dr}$. $\mathrm{H}$. Grossmann of DoDuco, Pforzheim, in a paper on "The Application of Precious Metal Deposits on Connectors". Tests were reported on the friction and wear of commercial connectors of both the plug and socket and the blade and spring contact types having hard gold deposits with varying contents of nickel and cobalt. 'The insertion force in the case of nickel hardened gold showed a steep drop at around 2.5 atomic per cent, remaining fairly constant with higher nickel contents. Similar results were obtained with cobalt-gold, the minimum value of insertion force occurring at 1.6 atomic per cent cobalt.

The contact resistance results showed that after a stable period up to 1000 insertions the hard gold deposits gave an abrupt increase in contact resistance, while pure gold showed an increase at an earlier stage. The amount of wear was of course markedly less with the hard golds than with pure gold, the wear behaviour being basically quite different with only small nickel contents.

A second part of the paper reported a comparative study of palladium, ruthenium and rhodium electrodeposits with the hard golds. Using 2 to 3 microns of noble metal on nickel it was found that both ruthenium and rhodium showed substantial increases in contact resistance after only a low number of operations, although the amount of wear was very small. Palladium showed very much less wear, but the contact resistances were also much higher than with hard gold. Scanning electron microscopy showed that while palladium deposits were subjected to plastic deformation, ruthenium and rhodium showed deep scratches down to the nickel barrier layer caused by very small but extremely hard and abrasive particles of the deposited metals, and leading of course to contact failure.

The author concluded that palladium electrodeposits might be considered as an alternative to hard gold in some circumstances but that with low contact pressures hard gold remains the most reliable contact material.

L. B. H. 